\title{
Particle acceleration via converter mechanism
}

\author{
Evgeny V. Derishev ${ }^{1,2}$, Vitaly V. Kocharovsky ${ }^{1,3}$, \\ Vladimir V. Kocharovsky ${ }^{1}$ and Felix A. Aharonian ${ }^{2}$ \\ ${ }^{1}$ Institute of Applied Physics RAS, Nizhny Novgorod, Russia \\ email: kochar@appl.sci-nnov.ru \\ ${ }^{2}$ Max-Planck-Institut für Kernphysik, Heidelberg, Germany \\ ${ }^{3}$ Texas A\&M University, College Station, TX, USA
}

\begin{abstract}
We review the problem of particle acceleration in relativistic shocks or shear flows. We propose a converter mechanism, which operates via continuous conversion of accelerated particles from charged into neutral state and back, and show that it is capable of producing the highest energy cosmic rays.
\end{abstract}

Keywords. Relativistic jets and shock waves, acceleration of particles, high-energy cosmic rays

We compare different acceleration mechanisms and show that the converter mechanism, suggested recently (see Derishev et al. 2003), is the least sensitive to the geometry of the magnetic field in accelerators and can routinely operate up to cosmic-ray energies close to the fundamental limit. The converter mechanism utilizes multiple conversions of charged particles into neutral ones (protons to neutrons and electrons/positrons to photons) and back by means of photon-induced reactions or inelastic nucleon-nucleon collisions. It works most efficiently in relativistic shocks or shear flows under the conditions typical for Active Galactic Nuclei, Gamma-Ray Bursts, and microquasars, where it outperforms the standard diffusive shock acceleration. The main advantages of the converter mechanism in such environments are that it greatly diminishes particle losses downstream and avoids the reduction in the energy gain factor, which normally takes place due to highly collimated distribution of accelerated particles.

Paradoxically, interactions with photons, which have been always treated as dissipative processes leading to degradation of particle energy, in this scenario play a positive role: they allow (through the charge-changing particle conversion, e.g., via photopionic reactions) the maximum energy gain to remain up to the largest particle energies. Indeed, a particle in the neutral state can freely cross the magnetic field lines, which allows it to avoid both particle losses downstream and reduction in the energy gain factor, which normally takes place due to highly collimated distribution of accelerated particles.

We also analyze the properties of gamma-ray radiation, which accompanies acceleration of particles via the converter mechanism and can provide evidence for the latter. In particular, we point out the fact that the opening angle of the radiation beam-pattern is different at different photon energies, which is relevant to the observability of the cosmicray sources as well as to their timing properties. Various consequences for observations are discussed.

\section{Reference}

Derishev, E. V., Aharonian, F. A., Kocharovsky, V. V., \& Kocharovsky, Vl. V. 2003, Phys. Rev. D, 2003, 68, 043003 